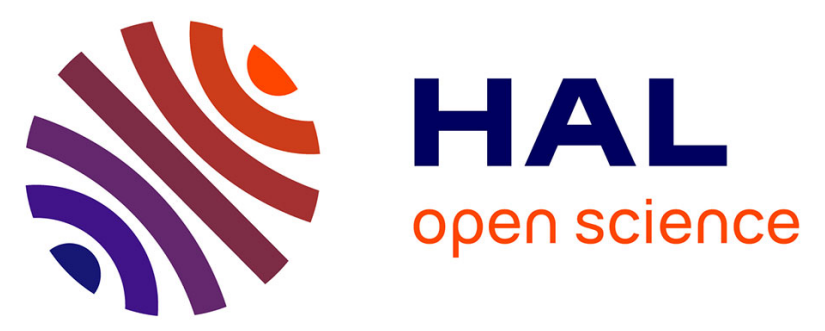

\title{
Méningiomes de la base du crâne: efficacité clinique et radiologique basée sur une analyse volumétrique quantitative
}

Y. Brahimi, D. Antoni, R. Srour, P. Wagner, F. Proust, A. Thiery, A. Labani, G. Noël

\section{To cite this version:}

Y. Brahimi, D. Antoni, R. Srour, P. Wagner, F. Proust, et al.. Méningiomes de la base du crâne: efficacité clinique et radiologique basée sur une analyse volumétrique quantitative. Cancer/Radiothérapie, 2019, 23, pp.290 - 295. 10.1016/j.canrad.2018.11.005 . hal-03486432

\section{HAL Id: hal-03486432 \\ https://hal.science/hal-03486432}

Submitted on 20 Dec 2021

HAL is a multi-disciplinary open access archive for the deposit and dissemination of scientific research documents, whether they are published or not. The documents may come from teaching and research institutions in France or abroad, or from public or private research centers.
L'archive ouverte pluridisciplinaire HAL, est destinée au dépôt et à la diffusion de documents scientifiques de niveau recherche, publiés ou non, émanant des établissements d'enseignement et de recherche français ou étrangers, des laboratoires publics ou privés.

\section{(c) (1) $\$$}

Distributed under a Creative Commons Attribution - NonCommerciall 4.0 International 
Méningiomes de la base du crâne : efficacité clinique et radiologique basée sur une analyse volumétrique quantitative

Skull base meningioma: clinical and radiological efficacy based on a quantitative volumetric analysis

Youssef BRAHIMI ${ }^{1}$; Delphine ANTONI ${ }^{1,2}$ MD, MSc; Robin SROUR ${ }^{3}$, MD; Pierre WAGNER $^{4}, \mathrm{MD}$, François PROUST ${ }^{5}, \mathrm{MD}, \mathrm{PhD}$; Alicia THIERY ${ }^{6}$ MD; Aïssam LABANI ${ }^{7}$, MD; Georges NOEL ${ }^{1,2^{*}}, \mathrm{MD}, \mathrm{PhD}$

1- University Radiation Oncology Department, Comprehensive Cancer Center Paul Strauss, 3, rue de la porte l'hôpital F-67065 Strasbourg Cedex

2- Laboratory of radiobiology, EA 3430, Federation of Translational medicine, Strasbourg (FMTS), Strasbourg University, F-67000 Strasbourg, France

3- Neurosurgery Department, hôpital Pasteur, 39 avenue de la liberté, F-6800 Colmar

4- Departement of Radiology, Comprehensive Cancer Center Paul Strauss, 3, rue de la porte l’hôpital F-67065 Strasbourg Cedex

5- Neurosurgery Department, University hospital of Strasbourg, 1 rue Molière, F-67000 Strasbourg

6- Epidemiology and biostatistics department, Comprehensive Cancer Center Paul Strauss, 3, rue de la porte l'hôpital F-67065 Strasbourg Cedex

7- Departement of Radiology, University hospital of Strasbourg, 1 Place de l'Hôpital, 67000 Strasbourg

* Correspondance: $\operatorname{Pr}$ Georges NOEL, MD, $\mathrm{PhD}$, same address, gnoel@strasbourg.unicancer.fr

\# de mots : 4062 


\section{\# de mots hors première page, résumés et références : 1967}

\section{résumés mots clés : 512}

\section{Résumé :}

Objectif de l'étude : recherche d'une corrélation entre efficacité clinique et radiologique basée sur un suivi volumétrique quantitatif après irradiation de méningiomes de la base du crâne.

Matériel et méthodes: Les données de 35 patients pris en charge soit par tomothérapie hélicoïdale $(45,7 \%)$ soit par radiothérapie fractionnée en conditions stéréotaxiques $(54,3 \%)$ ont été rétrospectivement analysées. Il s'agissait principalement de femmes (94\%) de 59 ans (43 - 81) avec des lésions du sinus caverneux (60\%). Il y avait une médiane de 2 (1 - 4) symptômes par patient, principalement des troubles visuels (39\%), des déficits des nerfs crâniens $(23,4 \%)$ et les céphalées $(17,2 \%)$.

Résultats: Le volume tumoral avait significativement diminué ( $\mathrm{p}<0,05)$ passant d'une médiane de $9.6 \mathrm{~mL}(0.3$ - 36.6) à $6.8 \mathrm{~mL}(0.1$ - 26.5) après un suivi médian de 44 mois $(24$ 77). Une amélioration d'au moins un symptôme a été retrouvée chez $63 \%$ des patients. En analyse unifactorielle, l'efficacité clinique $(p<0,05)$, la technique de radiothérapie $(p<0,05)$, la topographie tumorale $(\mathrm{p}<0,05)$ et le volume tumoral initial $(\mathrm{p}<0,05)$ étaient des facteurs prédictifs de la réponse radiologique. En analyse multifactorielle, seule la corrélation inverse entre réponse radiologique et volume tumoral initial demeurait significative ( $\rho$ : $-0,47$; intervalle e confiance à $95 \%: 3,2-5,7 ; \mathrm{p}<0,05)$.

Conclusion : Le suivi volumétrique quantitatif permet de mettre en évidence une efficacité radiologique majeure de la radiothérapie. Cependant, aucune corrélation franche entre efficacité clinique et radiologique n'a été retrouvée.

Mots clés: méningiome, base du crâne, radiothérapie stéréotaxique, RCMI, résultat thérapeutique, analyse volumétrique 
Abstract:

Purpose: To date, no correlation has been found between clinical and radiological efficacy after irradiation of skull base meningiomas. However, the evaluation of the radiological response was most often made by questionable methods that may have underestimated the radiological effectiveness of radiotherapy. The objective of this work is to verify this hypothesis by quantitative volumetric analysis.

Material and methods: Data from 35 patients treated with either helical tomotherapy (45.7\%) (TH) or fractionated stereotactic radiotherapy (RFCS) (54.3\%) (CSFR) were retrospectively analyzed. These were mainly women (94\%) aged 59 (43 - 81) with lesions mainly of the cavernous sinus $(60 \%)$. There was a median of $2(1-4)$ symptoms and the main symptoms were visual impairment (39\%), cranial nerve deficits (DNC) (23.4\%) and headaches (17.2\%). Results: Median tumor volume decreased significantly $(\mathrm{p}<0.05)$ from $9.6 \mathrm{~mL}(0.3-36.6)$ to $6.8 \mathrm{~mL}(0.1$ - 26.5) after median follow-up of 44 months $(24-77)$. Sixty-three percent of patients had an improvement of at least one symptom. In univariate analysis, clinical efficacy ( $\mathrm{p}<0.05)$, radiotherapy technique $(\mathrm{p}<0.05)$, tumor topography $(\mathrm{p}<0.05)$ and initial tumor volume $(\mathrm{p}<0.05)$ were predictive factors for radiological response. In multivariate analysis, only the inverse correlation between radiological response and initial tumor volume remained significant ( $\rho:-0.4795 \%$ CI -3.2 to $5.7 ; \mathrm{p}<0.05)$.

Conclusion: The quantitative volumetric monitoring demonstrates a major radiological efficiency of radiotherapy. However, no clear correlation between clinical and radiological efficacy was found.

Key words: meningioma, skull base, stereotactic radiation, intensity modulated radiation therapy, volumetric analyzes 


\section{Introduction}

L'objectif principal de la prise en charge des méningiomes de la base du crâne est la préservation neurologique combinée au contrôle local [1-6]. Le traitement de référence des méningiomes est l'exérèse tumorale radicale [7]. Cependant, dans le cas des lésions de la base du crâne, les taux de morbidité et de mortalité associée à ce geste peuvent atteindre respectivement, $67 \%$ et $8,3 \%[3,8]$ et ce même malgré le recours aux techniques les plus modernes de microchirurgie et même si le geste est effectué par un chirurgien expérimenté $[1,3,9,10]$. Progressivement, l'objectif d'une exérèse tumorale radicale a été abandonné au profit d'un geste chirurgical conservateur moins morbide [1,3,9-11]. Toutefois, l'exérèse tumorale partielle pourrait majorer le risque de récidive tumorale [2,12-14].

Bien qu'initialement discutée, la radiothérapie s'est progressivement imposée comme traitement adjuvant voire exclusif des méningiomes de la base du crâne [15-24]. Si l'efficacité et la tolérance clinique acceptables de la radiothérapie ne semblent actuellement plus à démontrer, des questions subsistent quant au mécanisme physiologique des radiations ionisantes. En effet, il y a une dissociation entre les faibles taux de réduction tumorale rapportés (20\% en moyenne) et les taux satisfaisants d'efficacité clinique rapportés après irradiation de méningiomes de la base du crâne (environ 46\%) [15-17,19,21,23-30].

Si la notion de dissociation clinico-radiologique [19,26,29,30] est dorénavant admise, des hypothèses tentant d'expliquer l'efficacité clinique de la radiothérapie par d'autres mécanismes que la décompression tumorale ont été proposées [16,30]. Cependant, la majorité des études qui ont rapporté l'efficacité radiologique n'ont soit pas détaillé les critères d'évaluation de celle-ci ou soit se sont basés sur des critères discutables d'évaluation [17,19,23,28,30-36]. 
Généralement, les critères RECIST (Response Evaluation Criteria in Solid Tumors)sont la référence pour évaluer la réponse radiologique après une thérapie antitumorale [37,38]. Cependant, du fait de la réponse tumorale lente associée à la supposée radiorésistance des méningiomes, la méthode RECIST n'est peut être pas adaptée pour le suivi radiologiques post-irradiation des méningiomes [4,5]. Une hypothèse est que les modifications de diamètres pourraient être trop subtiles pour pouvoir être détectées par des mesures unidimensionnelles.

Cette hypothèse est confortée par les résultats d'efficacité radiologique significativement plus élevés dans les études qui ont effectué un suivi volumétrique quantitatif rapportant un taux de régression tumorale de 78,5\% (33,2 - 100) [4-6,39-45]. Cependant, malgré des taux de régression tumorale se rapprochant des taux d'efficacité clinique, aucun suivi volumétrique quantitatif n'a mis en évidence de corrélation entre réponse clinique et radiologique [40,42].

Cette étude s'est proposée d'étudier la corrélation entre l'évolution volumétrique de 35 méningiomes de la base du crâne après irradiation fractionnée en conditions stéréotaxiques ou tomothérapie hélicoïdale et l'efficacité clinique.

\section{Matériel et méthodes}

De janvier 2009 à janvier 2014, 46 patients ont été pris en charge par radiothérapie pour un méningiome de la base du crâne. Onze patients ont été exclus en raison de données radiologiques non exploitables dans le cadre de cette étude. La plupart des patients étaient des femmes (94\%) et l'âge médian était de 59 ans (43 - 81). Les données cliniques sont résumées dans le tableau 1.

La radiothérapie était le traitement exclusif pour 18 patients, post opératoire pour 8 patients et de rattrapage pour 9 patients. La radiothérapie de rattrapage concernait des patients qui avaient initialement bénéficié d'une exérèse tumorale incomplète du méningiome et pour lesquels la suite de prise en charge avait consisté en une surveillance simple. Lorsqu'une 
majoration du volume tumoral résiduel postopératoire avait été constatée, l'indication de radiothérapie de rattrapage avait été retenue. Les critères de décision entre radiothérapie adjuvante immédiate ou de rattrapage n'étaient pas précisés.

Les patients ont été pris en charge soit par irradiation fractionnée en conditions stéréotaxiques $(54,3 \%)$ soit par tomothérapie hélicoïdale $(45,7 \%)$. La dose prescrite était de 54 Gy en 30 fractions de 1,8 Gy, sauf pour un patient avec une invasion osseuse confirmée histologiquement qui a reçu 60 Gy en 30 fractions de 2 Gy. La majorité des lésions étaient au niveau du sinus caverneux $(60 \%)$.

Seulement deux patients étaient asymptomatiques. Les patients restants avaient une médiane de $2(1$ - 4) symptômes par patient pour un total de 63 symptômes. Les principaux symptômes étaient les troubles visuels (38\%), les déficits des nerfs crâniens (24\%) et les céphalées $(17,5 \%)$.

La décision de la prise en charge par irradiation fractionnée en conditions stéréotaxiques ou tomothérapie hélicoïdale a été discutée au cours d'un comité technique. Les patients étaient immobilisés avec un masque de contention thermoformé adapté à la technique d'irradiation prévue. Le scanner dosimétrique était réalisé en position de traitement. Une IRM à visée dosimétrique a été effectuée en séquence T1 3D MPR (multiplanar reconstruction) avec injection de gadolinium et en séquence T2.

Afin de délinéer les volumes cibles et les organes à risques, le scanner et l'IRM ont été transférés et recalés dans le logiciel iPlan RT Image 4.1.2 (BrainLAB®, Feldkirchen, Germany) pour les patients pris en charge par irradiation fractionnée en conditions stéréotaxiques ou dans le logiciel FocalSim (CMS Focus, St Louis, MO) pour ceux pris en charge par tomothérapie hélicoïdale. 
Pour les patients pris en charge par irradiation fractionnée en conditions stéréotaxiques, la dose était délivrée par un accélérateur linéaire Novalis TX® (Varian Medical Systems, Palo Alto, CA, USA and BrainLAB®, Feldkirchen, Germany). Les autres patients ont été traités sous une tomothérapie HiArt ou HD (Accuray Inc. Sunnyvale, Californie, Etats-Unis).

Le suivi médian était de 44 mois (24 - 77). Les patients avaient une IRM 6 mois après la radiothérapie puis tous les ans. Au total, 206 IRM étaient disponibles.

L'évaluation de la réponse radiologique globale était basée sur une comparaison entre la mesure quantitative effectuée sur l'IRM initiale et celle sur la dernière IRM. Une réduction du volume tumoral était considérée comme une réponse partielle. Pour effectuer une analyse de la cinétique tumorale, des points de contrôle du suivi radiologique ont été définis 6 mois après la radiothérapie puis tous les 12 mois. Le nombre d'IRM disponibles 6, 12, 24, 36, 48 et 60 mois après la fin de la radiothérapie était respectivement de 17, 18, 27, 26, 16 et 9. La réponse radiologique globale et la cinétique tumorale ont été calculées en valeurs absolues et relatives.

Les logiciels de radiothérapie évaluent avec précision le volume délinéés [5,40,40,42,45]. Toutes les IRM ont été transférées vers un logiciel de radiothérapie Artiview (Aquilab®, Loos, France) afin d'effectuer la délinéation systématique des méningiomes sur les IRM initiales puis sur les IRM de surveillance. Les limites de la prise de contraste sur chaque coupe d'IRM T1 3 D MPR gado étaient considérées comme étant les marges tumorales et étaient délinées par le même opérateur (YB). Le volume tumoral était ensuite déterminé par le logiciel [46]. Au total, 151 IRM ont pu être exploitées par le logiciel de mesure soit une moyenne de 4,3 IRM par patient $(1-6)$. 
La radiothérapie a été considérée comme efficace dans les cas d'amélioration au moins partielle d'au moins un symptôme sans aggravation concomitante d'un autre symptôme ni nouveau symptôme.

La recherche d'une corrélation entre la réponse radiologique et le volume tumoral initial, la topographie tumorale, la technique de radiothérapie et l'efficacité clinique a été effectuée. Les analyses statistiques ont été effectuées avec le logiciel R. Un résultat était considéré significatif si $\mathrm{p}<0,05$.

\section{Résultats}

Il y avait une amélioration d'au moins un symptôme chez $63 \%$ des patients (tableau 2). Il y avait une amélioration au moins partielle de 49,2\% des symptômes initiaux. Les troubles visuels, les déficits des nerfs crâniens et les céphalées ont été les symptômes les plus améliorés dans respectivement $54,2 \%, 46,7 \%$ et $45,5 \%$ des cas. Le délai médian d'amélioration clinique était de 16,6 mois $(4,9$ - 48,3).

Lors du dernier suivi seul deux patients n'avaient pas de réduction tumorale. Le volume tumoral médian initial et au dernier suivi étaient respectivement de 9,6 $\mathrm{mL}(0,3-$ $36,6)$ et $6,8 \mathrm{~mL}(0,1-26,5)(\mathrm{p}<0,05)$. Le volume avait varié de $-83,9 \%$ à $+2 \%$ (médiane 29,4\%), ce qui correspondait à une décroissance absolue de $-31 \mathrm{~mL}$ à $0 \mathrm{~mL}$ (médiane $-3 \mathrm{~mL}$ ) ( $\mathrm{p}<0.05)$. A l'issue du suivi, le taux de réponse partielle était de $94 \%$. En se basant sur les comptes rendus radiologiques, le taux de réponse partielle était de $12 \%$.

A 6, 12, 36, et 60 mois la réduction absolue du volume était de respectivement de $1,03 \mathrm{ml}$ (SD [Dévaiation standard] : 1,05), -2,3 $\mathrm{ml}(\mathrm{SD}: 1,8),-3,9 \mathrm{ml}$ (SD : 5,1), et -7,3 ml $(\mathrm{SD}: 9,2)(p<0,05)$ (figures 1 et 2$)$.

En analyse unifactorielle, la réponse radiologique globale était inversement corrélée avec volume tumoral initial (Coefficient Rho de Spearman $=-0,78 ; p=0,05$ ). 
Il apparaissait une diminution significative du volume tumoral en cas d'efficacité clinique, de lésion du sinus caverneux et de tomothérapie. Cependant, dans ces 3 cas, les lésions étaient significativement plus larges chez les patients qui avaient ces caractéristiques que chez ceux qui ne les avaient pas, respectivement 15,4 $\mathrm{mL}(1,4-36,6)$ et $9,2(0,4-8,7)$ (p $<0,05), 8 \mathrm{~mL}(6,3-36,6)$ et $6,6 \mathrm{~mL}(0,3-16,8)(\mathrm{p}<0,05)$ et enfin $20 \mathrm{~mL}(3,2-36,6)$ et 8,4 $\mathrm{mL}(0,3-24,9)(\mathrm{p}<0,05)$

En analyse multifactorielle, seul le volume tumoral initial conservait une corrélation inverse statistiquement significative avec la réponse radiologique ( $\rho:-0.47$ 95\% CI -3.2 to 5.7; $\mathrm{p}<0.05)($ tableau 3).

Il n’y avait pas de corrélation directe entre réponses clinique et radiologique. A signaler cependant que parmi les patients avec une amélioration clinique il y avait $95 \%$ de réponse radiologique contre $87,5 \%$ pour les autres $(\mathrm{p}<0,05)$

\section{DISCUSSION}

Un taux de $94 \%$ de réponse tumorale partielle a été retrouvé. Ce taux est comparable avec les résultats rapportés par d'autres études volumétriques [4,5,42,44]. Ce taux est largement supérieur aux taux de réponse radiologique rapportés par les études qui ont évalué la reponse radiologique sur la base d'une simple description qualitative ou d'une diminution du plus grand diamètre tumoral [15-17,19,21,23-30]. Cette étude illustre parfaitement la différence en fonction des évaluations radiologiques en comparant les $12 \%$ de réponse tumorale partielle sur les derniers comptes-rendus radiologiques de suivi et les $94 \%$ de réduction du volume tumoral par l'étude volumétrique quantitative.

Ainsi la notion de dissociation clinico-radiologique rapportée après irradiation des méningiomes de la base du crane pourrait surtout être due à une sous-estimation de l'efficacité radiologique [47]. Toutefois cette étude, comme d'autres précédemment, n'a pas 
permis de mettre en évidence une corrélation entre la réduction volumétrique et la réponse clinique $[40,42]$. Il est notable, que la réponse clinique était corrélée au volume tumoral initial. Or, ce volume est aussi prédictif de la réponse radiologique. Ainsi, l'absence de corrélation directe en réponse tumorale et réponse clinique est possiblement due à ce facteur confondant. Il s'agit donc de la première étude qui retrouve le volume tumoral initial élevé comme facteur prédictif positif de décroissance tumorale après radiothérapie. Des résultats contraires ont été signalés dans d'autres études [5,42,45]. Mais ces résultats sont difficiles à comparer avec les autres séries car il existe trop de disparités dans ces études [4-6,40-45].

Afin d'observer une réduction conséquente des volumes, un suivi long est nécessaire. La tendance à la stabilisation tumorale a été signalée entre 6 et 8 ans de suivi [44], même si dans notre étude, aucun patient ne rechute après 36 mois.

La principale limite de cette série est l'absence de cas de progression tumorale avérée pour avoir un comparatif volumétrique quantitatif et de la cinétique tumorale avec les $94 \%$ de régression tumorale retrouvés. Il faut également prendre en compte qu'il s'agit d'une analyse rétrospective concernant un faible effectif avec un suivi relativement court.

\section{CONCLUSION}

La dissociation clinico-radiologique signalée après irradiation des méningiomes de la base du crâne pourrait en fait être due à une sous-estimation de l'efficacité radiologique. L'évaluation radiologique devrait systématiquement être effectuée pas des analyses volumétriques quantitatives. Cependant, d'autres études sont nécessaires pour démontrer qu'il existe une corrélation entre efficacité clinique et réduction du volume tumoral. 


\section{REFERENCES:}

[1] Nanda A, Thakur JD, Sonig A, Missios S. Microsurgical resectability, outcomes, and tumor control in meningiomas occupying the cavernous sinus. J Neurosurg 2016;125:378-92

[2] Almefty R, Dunn IF, Pravdenkova S, Abolfotoh M, Al-Mefty O. True petroclival meningiomas: results of surgical management. J Neurosurg 2014;120:40-51

[3] Pichierri A, Santoro A, Raco A, Paolini S, Cantore G, Delfini R. CAVERNOUS SINUS MENINGIOMAS: RETROSPECTIVE ANALYSIS AND PROPOSAL OF A TREATMENT ALGORITHM. Neurosurgery 2009;64:1090-101

[4] Mozes P, Dittmar JO, Habermehl D, Tonndorf-Martini E, Hideghety K, Dittmar A, et al. Volumetric response of intracranial meningioma after photon or particle irradiation. Acta Oncol 2017;56:431-7

[5] Astner ST, Theodorou M, Dobrei-Ciuchendea M, Auer F, Kopp C, Molls M, et al. Tumor Shrinkage Assessed by Volumetric MRI in the Long-Term Follow-Up after Stereotactic Radiotherapy of Meningiomas. Strahlenther Onkol 2010;186:423-9

[6] Park YG, Kim EY, Chang JW, Chung SS. Volume changes following gamma knife radiosurgery of intracranial tumors. Surg Neurol 1997;48:488-93.

[7] Simpson D. The recurrence of intracranial meningiomas after surgical treatment. J Neurol Neurosurg Psychiatry 1957;20:22-39.

[8] Li D, Tang J, Ren C, Wu Z, Zhang L-W, Zhang J-T. Surgical management of medium and large petroclival meningiomas: a single institution's experience of 199 cases with long-term follow-up. Acta Neurochir (Wien) 2016;158:409-25 
[9] Couldwell WT, MacDonald JD, Taussky P. Complete Resection of the Cavernous SinusIndications and Technique. World Neurosurg 2014;82:1264-70

[10] Walsh MT, Couldwell WT. Management options for cavernous sinus meningiomas. J Neurooncol 2009;92:307-16

[11] Sindou M, Wydh E, Jouanneau E, Nebbal M, Lieutaud T. Long-term follow-up of meningiomas of the cavernous sinus after surgical treatment alone. J Neurosurg 2007;107:937-44

[12] De Jesús O, Sekhar LN, Parikh HK, Wright DC, Wagner DP. Long-term follow-up of patients with meningiomas involving the cavernous sinus: recurrence, progression, and quality of life. Neurosurgery 1996;39:915-919-920.

[13] Cusimano MD, Sekhar LN, Sen CN, Pomonis S, Wright DC, Biglan AW, et al. The results of surgery for benign tumors of the cavernous sinus. Neurosurgery 1995;37:1-9-10.

[14] DeMonte F, Smith HK, al-Mefty O. Outcome of aggressive removal of cavernous sinus meningiomas. J Neurosurg 1994;81:245-51

[15] Milker-Zabel S, Zabel-du Bois A, Huber P, Schlegel W, Debus J. Fractionated Stereotactic Radiation Therapy in the Management of Benign Cavernous Sinus Meningiomas: Long-Term Experience and Review of the Literature. Strahlenther Onkol 2006;182:635-40

[16] Pirzkall A, Debus J, Haering P, Rhein B, Grosser K-H, Höss A, et al. Intensity modulated radiotherapy (IMRT) for recurrent, residual, or untreated skull-base meningiomas: preliminary clinical experience. Int J Radiat Oncol 2003;55:362-72 
[17] Brell M, Villà S, Teixidor P, Lucas A, Ferrán E, Marín S, et al. Fractionated stereotactic radiotherapy in the treatment of exclusive cavernous sinus meningioma: functional outcome, local control, and tolerance. Surg Neurol 2006;65:28-33

[18] Noel G, Renard A, Valéry C, Mokhtari K, Mazeron JJ. Rôle de la radiothérapie dans le traitement des méningiomes cérébraux. Cancer/Radiothérapie 2001;5:217-236.

[19] Noël G, Bollet MA, Calugaru V, Feuvret L, Haie-Meder C, Dhermain F, et al. Functional outcome of patients with benign meningioma treated by $3 \mathrm{D}$ conformal irradiation with a combination of photons and protons. Int J Radiat Oncol Biol Phys 2005;62:1412-1422.

[20] Milker-Zabel S, Zabel A, Schulz-Ertner D, Schlegel W, Wannenmacher M, Debus J. Fractionated stereotactic radiotherapy in patients with benign or atypical intracranial meningioma: Long-term experience and prognostic factors. Int J Radiat Oncol 2005;61:809-16

[21] Milker-Zabel S, Zabel-du Bois A, Huber P, Schlegel W, Debus J. Intensity-Modulated Radiotherapy for Complex-Shaped Meningioma of the Skull Base: Long-Term Experience of a Single Institution. Int $\mathbf{J}$ Radiat Oncol 2007;68:858-63

[22] Combs SE, Adeberg S, Dittmar J-O, Welzel T, Rieken S, Habermehl D, et al. Skull base meningiomas: Long-term results and patient self-reported outcome in 507 patients treated with fractionated stereotactic radiotherapy (FSRT) or intensity modulated radiotherapy (IMRT). Radiother Oncol 2013;106:186-91

[23] Litré CF, Colin P, Noudel R, Peruzzi P, Bazin A, Sherpereel B, et al. Fractionated Stereotactic Radiotherapy Treatment of Cavernous Sinus Meningiomas: A Study of 100 Cases. Int J Radiat Oncol 2009;74:1012-7 
[24] Soldà F, Wharram B, De Ieso PB, Bonner J, Ashley S, Brada M. Long-term efficacy of fractionated radiotherapy for benign meningiomas. Radiother Oncol 2013;109:330-4

[25] Correa SFM, Marta GN, Teixeira MJ. Neurosymptomatic carvenous sinus meningioma: a 15-years experience with fractionated stereotactic radiotherapy and radiosurgery. Radiat Oncol Lond Engl 2014;9:27

[26] Shen X, Andrews DW, Sergott RC, Evans JJ, Curran WJ, Machtay M, et al. Fractionated stereotactic radiation therapy improves cranial neuropathies in patients with skull base meningiomas: a retrospective cohort study. Radiat Oncol 2012;7:225

[27] Onodera S, Aoyama H, Katoh N, Taguchi H, Yasuda K, Yoshida D, et al. Long-term Outcomes of Fractionated Stereotactic Radiotherapy for Intracranial Skull Base Benign Meningiomas in Single Institution. Jpn J Clin Oncol 2011;41:462-8

[28] Minniti G, Amichetti M, Enrici RM. Radiotherapy and radiosurgery for benign skull base meningiomas. Radiat Oncol Lond Eng1 2009;4:42

[29] Metellus P, Batra S, Karkar S, Kapoor S, Weiss S, Kleinberg L, et al. Fractionated Conformal Radiotherapy in the Management of Cavernous Sinus Meningiomas: Long-Term Functional Outcome and Tumor Control at a Single Institution. Int J Radiat Oncol 2010;78:836-43

[30] Selch MT, Ahn E, Laskari A, Lee SP, Agazaryan N, Solberg TD, et al. Stereotactic radiotherapy for treatment of cavernous sinus meningiomas. Int J Radiat Oncol 2004;59:101-11

[31] Hafez RFA, Morgan MS, Fahmy OM. Stereotactic Gamma Knife surgery safety and efficacy in the management of symptomatic benign confined cavernous sinus meningioma. Acta Neurochir (Wien) 2015;157:1559-64 
[32] Starke R, Kano H, Ding D, Nakaji P, Barnett GH, Mathieu D, et al. Stereotactic radiosurgery of petroclival meningiomas: a multicenter study. J Neurooncol 2014;119:169-76

[33] Pollock BE, Stafford SL, Link MJ, Garces YI, Foote RL. Single-fraction radiosurgery of benign cavernous sinus meningiomas: Clinical article. J Neurosurg 2013;119:675-82

[34] Skeie BS, Enger P ø., Skeie GO, Thorsen F, Pedersen P-H. Gamma Knife Surgery of Meningiomas Involving the Cavernous Sinus: Long-term Follow-up of 100 Patients. Neurosurgery 2010;66:661-9

[35] Metellus P, Regis J, Muracciole X, Fuentes S, Dufour H, Nanni I, et al. Evaluation of Fractionated Radiotherapy and Gamma Knife Radiosurgery in Cavernous Sinus Meningiomas: Treatment Strategy: Neurosurgery 2005;57:873-86

[36] Schiappacasse L, Cendales R, Sallabanda K, Schnitman F, Samblas J. Preliminary Results of Helical Tomotherapy in Patients with Complex-Shaped Meningiomas Close to the Optic Pathway. Med Dosim 2011;36:416-22

[37] Eisenhauer EA, Therasse P, Bogaerts J, Schwartz LH, Sargent D, Ford R, et al. New response evaluation criteria in solid tumours: Revised RECIST guideline (version 1.1). Eur J Cancer 2009;45:228-47

[38] Therasse P, Arbuck SG, Eisenhauer EA, Wanders J, Kaplan RS, Rubinstein L, et al. New Guidelines to Evaluate the Response to Treatment in Solid Tumors. JNCI J Natl Cancer Inst 2000;92:205-16

[39] dos Santos MA, de Salcedo JBP, Gutiérrez Diaz JA, Calvo FA, Samblás J, Marsiglia H, et al. LongTerm Outcomes of Stereotactic Radiosurgery for Treatment of Cavernous Sinus Meningiomas. Int J Radiat Oncol 2011;81:1436-41

[40] Feigl GC, Bundschuh O, Gharabaghi A, Samii M, Horstmann GA. Volume reduction in meningiomas after gamma knife surgery. J Neurosurg 2005;102 Suppl:189-94. 
[41] Feigl GC, Samii M, Horstmann GA. Volumetric follow-up of meningiomas: a quantitative method to evaluate treatment outcome of gamma knife radiosurgery. Neurosurgery 2007;61:281-286-287

[42] Henzel M, Gross MW, Hamm K, Surber G, Kleinert G, Failing T, et al. Significant Tumorvolume Reduction Of Meningiomas Afterstereotactic Radiotherapy: Results Of A Prospective Multicenter Study. Neurosurgery 2006;59:1188-94

[43] Kano H, Park K-J, Kondziolka D, Iyer A, Liu X, Tonetti D, et al. Does Prior Microsurgery Improve or Worsen the Outcomes of Stereotactic Radiosurgery for Cavernous Sinus Meningiomas?: Neurosurgery $2013 ; 73: 401-10$

[44] Lee NK, Kim CY, Yoon WS, Chung YG, Lee NJ. Quantitative analysis of tumor volume reduction after three-dimensional conformal radiation therapy for intracranial meningiomas. $\mathrm{J}$ Neurooncol 2015;121:195-9

[45] Harrison G, Kano H, Lunsford LD, Flickinger JC, Kondziolka D. Quantitative tumor volumetric responses after Gamma Knife radiosurgery for meningiomas. J Neurosurg 2016;124:146-54

[46] Snell JW, Sheehan J, Stroila M, Steiner L. Assessment of imaging studies used with radiosurgery: a volumetric algorithm and an estimation of its error. J Neurosurg 2006;104:157-62

[47] Brahimi Y, Antoni D, Srour R, Proust F, Cebula H, Labani A, et al. [Base of the skull meningioma: Efficacy, clinical tolerance and radiological evaluation after radiotherapy]. Cancer Radiother J Soc Francaise Radiother Oncol 2018;22:264-86 
Figure 1: Courbes de cinétique tumorale

Figure 1: Tumor kinetics curves




Figure 2 : Réduction significative du volume tumoral : exemple d'une patiente

Figure 2: Significant tumor shrinkage: example of a patient

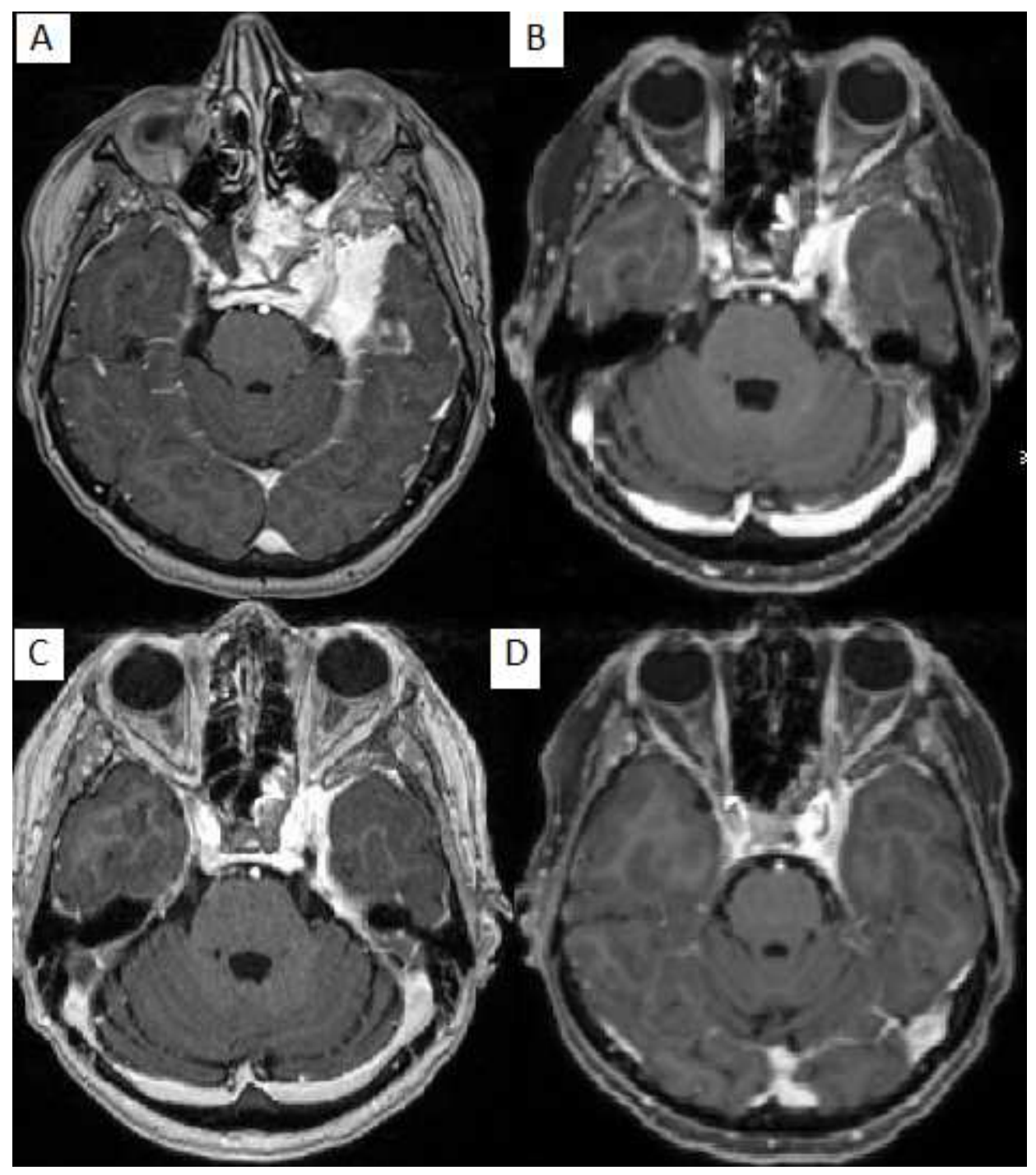

$\mathrm{A}=$ volume tumoral initial; $\mathrm{B}=$ volume tumoral à 36 mois de suivi; $\mathrm{C}=$ volume tumoral à 48 mois; $\mathrm{D}=$ volume tumoral à 60 mois

$A=$ tumor volume at baseline; $B=$ tumor volume after 36 months of follow-up; $C=$ tumor volume after 48 months of follow-up; $D=$ tumor volume after 60 months of follow-up 
Tableau 1: Caractéristiques des patients

Table 1: Patient characteristics

\section{Caracteristiques}

Nombre de patients (Total 35)

Sexe

Femmes

$33(94 \%)$

Hommes $2(6 \%)$

Délai de surveillance médian (mois) (min - max)

$44(24-77)$

Age médian (année) (min - max)

$59(43-81)$

Topographie tumorale

\begin{tabular}{l|l} 
Sinus caverneux & $21(60 \%)$
\end{tabular}

Angle ponto-cérébelleux $6(17 \%)$

Clival $4(11,5 \%)$

Autres $4(11,5 \%)$

Latéralité

\begin{tabular}{|r|l|}
\hline Gauche & $21(60 \%)$ \\
Droite & $12(34,3 \%)$ \\
\hline Bilatéral & 2 \\
\hline Volume tumoral median (min - max) & $9,6 \mathrm{ml}(0,3-36,6)$ \\
\hline Dernier traitement & \\
\hline Radiothérapie exclusive & $18(51,4 \%)$ \\
\hline Radiothérapie de rattrapage & $9(25,7 \%)$ \\
\hline Radiothérapie adjuvante & $8(22,9 \%)$ \\
\hline
\end{tabular}


Tableau 3: Réponse clinique

Table 3: Clinical response

\begin{tabular}{|c|c|c|c|c|c|c|c|c|c|}
\hline $\begin{array}{l}\text { Efficacité } \\
\text { clinique }\end{array}$ & $\begin{array}{l}\text { Récupération } \\
\text { Complète }\end{array}$ & $\begin{array}{l}\text { Délai médian (mois) } \\
\text { (min - max) }\end{array}$ & $\begin{array}{l}\text { Récupération } \\
\text { partielle }\end{array}$ & $\begin{array}{l}\text { Délai médian (mois) } \\
\text { (min - max) }\end{array}$ & Stabilité & Aggravation & $\begin{array}{l}\text { Délai médian (mois) } \\
\text { (min - max) }\end{array}$ & $\begin{array}{c}\text { Non } \\
\text { Communiqué }\end{array}$ & Total \\
\hline Céphalées & 1 & 12 & 4 & $17,6(9,2-45,7)$ & 3 & 0 & & 3 & 11 \\
\hline $\begin{array}{l}\text { Nausées et } \\
\text { vomissemen }\end{array}$ & 1 & 4,9 & 2 & $15,6(12-19,3)$ & 3 & 0 & & 1 & 7 \\
\hline Epilepsie & 1 & 30,5 & 0 & 0 & 0 & 0 & & 0 & 1 \\
\hline $\begin{array}{l}\text { Troubles } \\
\text { visuels }\end{array}$ & 8 & $17,8(4,9-42,4)$ & 5 & $18,1(12-48,3)$ & 6 & 1 & 18,7 & 4 & 24 \\
\hline $\begin{array}{l}\text { Déficit nerf } \\
\text { crânien }\end{array}$ & 6 & $13,6(5,3-25,4)$ & 1 & 13,4 & 6 & 1 & 31,6 & 1 & 15 \\
\hline $\begin{array}{l}\text { Troubles } \\
\text { auditifs }\end{array}$ & 0 & & 2 & $33,8(19,3-48,3)$ & 3 & 0 & & 0 & 5 \\
\hline Total & 17 & & 14 & & 21 & 2 & & 9 & 63 \\
\hline
\end{tabular}


Tableau 3: Facteurs prédictifs de réponse radiologique

Table 3: Predictive factors of radiological response

\begin{tabular}{|c|c|c|}
\hline \multicolumn{3}{|c|}{ Facteurs prédictifs de réponse radiologique } \\
\hline \multicolumn{3}{|c|}{ Analyses univariées ( $\mathbf{p}<0.05)$} \\
\hline Efficacité clinique & En cas d'amélioration clinique $\mathbf{- 3 , 1} \mathbf{m L}(-30,7$ à $-0,3)$ & Pas d'amélioration clinique $\mathbf{- 2 , 1} \boldsymbol{m L}(-3$ à 0,2$)$ \\
\hline Technique de traitement & TH : $\mathbf{- 3 , 4} \boldsymbol{m L}(-30,7$ à 1,2$)$ & RFCS : $-\mathbf{2} \boldsymbol{m} \boldsymbol{L}(-12,7$ à 0,2$)$ \\
\hline Topographie tumorale & Sinus caverneux : $\mathbf{- 3 , 4} \boldsymbol{m L}(-30,7$ à $-1,2)$ & Autres localisations : $\mathbf{- 1 , 5} \mathbf{m L}(-9,9$ à 0,2$)$ \\
\hline Volume tumoral initial & En cas de lésion > 9,6 mL : $-4,3 m L(-31$ à 1$)$ & En cas de lésion $\leq 9,6 \mathrm{~mL}:-1,8 \mathrm{~mL}(-5$ à 0$)$ \\
\hline \multicolumn{3}{|c|}{ Analyses multivariées $(p<0.05)$} \\
\hline Volume tumoral initial & \multicolumn{2}{|c|}{ Corrélation inverse au volume tumoral initial ( $\rho$ : -0.47 95\%CI -3.2 à 5.7) } \\
\hline
\end{tabular}

